\title{
PESQUISA BIBLIOGRÁFICA SOBRE IMPLEMENTAÇÃO DA AUTOMAÇÃO NA PISCICULTURA
}

BIBLIOGRAPHIC RESEARCH ON IMPLEMENTATION OF AUTOMATION IN PISCICULTURE

\section{Matheus de Jesus Amorim ${ }^{*}$, Gabriéla Ramalho Sousa ${ }^{2}$, \& Marielce de Cássia Ribeiro Tosta $^{3}$}

\author{
${ }^{12} 3$ Centro Universitário Norte do Espírito Santo da Universidade Federal do Espírito Santo. \\ 1* amorim.matheus43@gmail.com² gabi.ramalho01@gmail.com ${ }^{3}$ marielcetosta@gmail.com
}

\section{ARTIGO INFO.}

\section{Recebido em: 01.03.2021}

Aprovado em: 06.04.2021

Disponibilizado em: 25.05 .2021

\section{Palavras-chave:}

Piscicultor; Produtividade; Qualidade; Automação.

\section{KEYWORDS:}

Fish farmer; Productivity; Quality; Automation.

*Autor Correspondente: Amorim, M. de J.

\section{RESUMO}

O uso das tecnologias de automação, podem melhorar a produção da aquicultura, de modo a favorecer a produtividade e qualidade do produto. $\mathrm{O}$ futuro deste comércio depende, principalmente, de como a indústria conduzirá o processo de automação de sua produção. Isto posto, objetivou-se neste estudo analisar o aumento da produtividade advinda com o uso da automação na alimentação e monitoramento da qualidade da água, além de descrever os projetos realizados com esta finalidade e as empresas que prestam tais serviços. Para isso, foi realizada revisão bibliográfica para levantar informações sobre os protótipos e, além disso, foi efetuado o contato com algumas empresas que fornecem o serviço de automação por completo ou parcialmente. Como resultado, foi possível notar que existem várias possibilidades de implementação da automação na piscicultura, variando os custos de implementação desde baixo a alto e conforme necessidade do piscicultor.

\begin{abstract}
The use of automation technologies, can improve aquaculture production, in order to favor productivity and product quality. The future of this trade depends mainly on how the industry will conduct the process of automating its production. That said, the objective of this study was to analyze the increase in productivity resulting from the use of automation in feeding and monitoring of water quality, in addition to describing the projects carried out for this purpose and the companies that provide such services. For this, a bibliographic review was carried out to gather information about the prototypes and, in addition, contact was made with some companies that provide the automation service in whole or in part. As a result, it was possible to notice that there are several possibilities for implementing automation in fish farming, varying the implementation costs from low to high and according to the fish farmer's needs.
\end{abstract}


Citação (APA): Amorim, M. de J., Sousa, G. R., \& Tosta, M. de C. R. (2021). Pesquisa bibliográfica sobre implementação da automação na piscicultura. Brazilian Journal of Production Engineering, 6(8), Edição Especial "Pesca e Aquicultura: Gestão, Política e Inovação", 85-96.

\section{INTRODUÇÃO}

Para contribuir na compreensão das mudanças ocorridas no setor do desenvolvimento e crescimento industrial, dois conceitos se destacam, sendo estes: a tecnologia e a técnica. No que tange a tecnologia, esta entende-se como o conjunto de teorias sobre os meios de produção, enquanto a técnica se refere à aplicação deste conjunto teórico na prática. Estes conceitos são empregados na produção de novos produtos, bem como nas mais diversas etapas das atividades produtivas, além de estarem associados à invenção e a inovação, essenciais para estimular o progresso tecnológico (Blaumer, 1964 apud Fleury, 1978; Longo, 1984).

Nesse sentido, a competição tecnológica se tornou a essência do desenvolvimento econômico por meio das três primeiras revoluções industriais, nas quais trouxeram produção em massa, linhas de montagem, eletricidade e tecnologia da informação, o que elevou a renda de trabalhadores. Quanto à quarta revolução industrial, esta se define por um conjunto de tecnologias que concedem a fusão do mundo físico, digital e biológico, sendo o seu impacto mais profundo e exponencial (Agência Brasileira de Desenvolvimento Industrial [ABDI], n.d.). Segundo Brettel, Friederichsen, Keller e Rosenberg (2014), este modelo colabora com a flexibilidade dos meios de produção, como também um complexo envolvimento na produção por intermédio de novas tecnologias empregadas que aumentam e facilitam o processo de difusão e lançamento de novos produtos no mercado. Desse modo, a integração converte-se em uma importante ferramenta para o aumento da produtividade das novas fábricas inteligentes.

Sob essa ótica, Schwab (2017) argumenta que a fusão de tecnologias nos domínios físico, digital e biológico tem o potencial de tornar fundamentalmente diferente a quarta revolução em relação às anteriores, e a transformação digital tem impactado os padrões de atividade, de interação humana e de produção em ritmo e escala sem precedentes. Assim, o reflexo das possibilidades proporcionadas pelas transformações do mundo digital são novas tecnologias, tais como: big data analytics, inteligência artificial, machine learning, cloud computing, internet das coisas (IoT) e manufatura 4.0.

Maciente, Rauen e Kubota (2019) definem os padrões da manufatura 4.0 como aqueles fundamentados na digitalização total dos processos produtivos, onde em uma mesma planta produtiva se associam componentes físicos e digitais. Nesse cenário estão incluídos entre os processos de digitalização: o embarcamento de sensores; a utilização de sistemas ciber físicos, de sistemas de segurança e de monitoramento de consumo energético; o emprego de sistemas analíticos e de monitoramento de dados relevantes para a produção. Com isso, por meio dessas novas aplicações de IoT, espera-se melhorias consideráveis nos processos industriais, como: uso de sistemas autônomos, nos quais permitirão maior produtividade no processamento de insumos, além de garantir maior precisão nos processos produtivos, sendo estes: uso de sensores que, ao realizar a captura de dados em tempo real, poderão prevenir eventuais problemas e garantir a segurança, bem como a eficiência energética e o bom funcionamento dos sistemas integrados; aplicações em manutenção preditiva, que poderão prever a necessidade de reposição de peças e eventuais desgastes, entres outras. $\mathrm{O}$ impacto da IoT na produtividade dos processos fabris pode gerar economia de $10 \%$ a $20 \%$ na utilização de recursos energéticos e de $10 \%$ a $25 \%$ na eficiência da mão de obra.

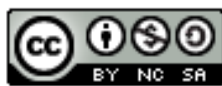


Citação (APA): Amorim, M. de J., Sousa, G. R., \& Tosta, M. de C. R. (2021). Pesquisa bibliográfica sobre implementação da automação na piscicultura. Brazilian Journal of Production Engineering, 6(8), Edição Especial "Pesca e Aquicultura: Gestão, Política e Inovação", 85-96.

Desta forma espera-se que a Indústria 4.0 potencialize maior agilidade e eficiência na produção e distribuição das mercadorias. O novo processo de produção industrial se tornaria mais inteligente e além disso, interligaria diversas tecnologias com intuito de aumentar sua eficiência produtiva e de abastecimento de matérias-primas, bem como de produtos nos mercados, o que levaria ao aumento da competitividade (Matos, 2018). O modo de organização da produção das companhias seria modificado através das tecnologias e, consequentemente, levaria à transformação da sua estrutura de organização e de planejamento (Danoesastro, Freeland \& Reichert, 2017).

Dentre as atividades econômicas no Brasil, a produção aquícola destaca-se e poderia se estabelecer como alternativa para enfrentar diversos problemas que atingem a produção mundial de alimentos, como: o potencial declínio da produção agropecuária consequente das mudanças climáticas; os efeitos negativos da tendência de alta dos preços das commodities agropecuárias no início do século XXI; e a volta da fome em vários países observado nos últimos anos (Siqueira, 2018).

Apesar de sua relevância, Schulter e Vieira (2017) ressaltaram que o ramo da piscicultura apresenta alguns fatores críticos quanto ao quesito tecnológico, nos quais atrapalham o seu desenvolvimento, como: a ausência de equipamentos específicos para a atividade; balanceamento de rações adequadas tanto para a espécie como para as regiões de produção, em virtude de que estas representam parte significativa dos custos de produção; uso de tanquesrede de grande volume; e adoção de sistemas produtivos mais intensivos.

No Brasil há alimentadores automáticos disponíveis no mercado, embora seu uso ainda seja limitado a um número restrito de empreendimentos devido ao seu elevado investimento. Brito, Pontes, Tsujii, Araújo e Ricther (2017) ressaltaram que os sistemas de automação podem ser empregados no ramo da piscicultura com o intuito de monitorar os parâmetros tanto físicos quanto químicos da qualidade da água de cultivo, bem como para ajustar e melhorar o fornecimento de ração, realizados por intermédio de softwares e/ou equipamentos próprios para essas finalidades, de modo a facilitar o manejo e proporcionar um ambiente em que os peixes possam apresentar seu máximo potencial produtivo.

As rações não podem permanecer por um longo período de tempo em contato com a água, uma vez que dessa forma perdem com rapidez grande parte dos seus nutrientes solúveis, tais como: proteínas e aminoácidos, carboidratos, vitaminas hidrossolúveis e minerais. Desta forma, os peixes não recebem a quantidade adequada de alimentação, o que acarreta em menor produtividade e maior tempo de cultivo, e consequentemente, maior custo de produção (Kubitza, 2019). Considerando a importância desse problema, este artigo teve como objetivo realizar uma revisão bibliográfica sobre o aumento da produtividade advinda com o uso da automação na alimentação e monitoramento da qualidade da água, além de descrever os projetos realizados com esta finalidade e as empresas que prestam tais serviços.

\section{Metodologia}

Para o presente artigo, foi realizado um estudo sobre automação na piscicultura e como esta pode influenciar no aumento da produtividade, através da pesquisa de referencial teórico. Para isso, o estudo foi dividido em duas vertentes, sendo a primeira relacionada a protótipos para 
Citação (APA): Amorim, M. de J., Sousa, G. R., \& Tosta, M. de C. R. (2021). Pesquisa bibliográfica sobre implementação da automação na piscicultura. Brazilian Journal of Production Engineering, 6(8), Edição Especial "Pesca e Aquicultura: Gestão, Política e Inovação", 85-96.

automatização, e a segunda às empresas que realizam prestação de serviço de automação ou comercializam produtos da área.

Os dados para elaboração da referida pesquisa foram coletados em artigos presentes no Google acadêmico no que tange aos protótipos, com relação às empresas foram realizados contatos com algumas por e-mail e ligação telefônica e por acesso aos sites das mesmas. Ademais, foi realizado um quadro comparativo entre as implementações de controladores.

Desse modo, após análise bibliográfica, foram levantados os principais meios para implementação da automação na piscicultura. Assim, a partir de tal metodologia, foi possível compreender a relevância deste estudo, bem como analisar diversos pontos existentes referente ao tema.

\section{Produtividade}

Para realizar a produção de peixes com qualidade Ostrensky e Boeger (1998) ressaltaram que existe uma periocidade diária necessária com a qual se deve analisar parâmetros como a temperatura da água, o oxigênio dissolvido e o pH para manter as características saudáveis da água. Assim, verifica-se que por meio de um processo manual, o piscicultor dispõe de kits de análise para medir tais parâmetros. Dessa forma, estes parâmetros devem ser medidos com maior frequência dada a periodicidade indicada na Tabela 1.

Tabela 1. Parâmetros e periodicidades para monitoramento da qualidade da água

\begin{tabular}{cc}
\hline Parâmetro & Periodicidade diária \\
\hline Temperatura da água & 2 \\
Oxigênio dissolvido & 2 \\
$\mathrm{pH}$ & 1 \\
\hline
\end{tabular}

Fonte: Ostrensky e Boeger (1998).

Por meio do emprego da automação, países desenvolvidos melhoraram seus índices zootécnicos e o manejo nas grandes pisciculturas intensivas (Lekang, 2009; Trabachini, 2013), e assim, fez com que a exploração seja cada vez mais competitiva e rentável (Suzuki \& Hernandez, 1999), porém, em território brasileiro a alimentação manual ainda é predominante.

$\mathrm{Na}$ indústria, tem-se que a "visão computacional" frequentemente vem sendo empregada, em contraste ao cenário encontrado na aquicultura, onde essa ainda é pouco utilizada, além de possuir limitações, devido a sensibilidade dos indivíduos inspecionados, que apresentam estresse facilmente e se movimentam para locais onde fatores como a luminosidade, visibilidade e estabilidade geralmente não são controláveis, e os sensores precisam operar submersos ou em ambiente úmido. Essa técnica tem potencial para ser aplicada em todas as fases de produção dos animais, tais como: cria, recria e engorda até a despesca, usada em tarefas como a de contagem, medida de tamanho e estimativa da biomassa, detecção de sexo e inspeção de qualidade, identificação de espécies e de estoques, bem como o monitoramento do bem-estar e do comportamento (Zion, 2012).

A alimentação é um dos maiores problemas da criação de peixes em tanques artificiais, pois essa precisa ser equilibrada e na quantidade correta para o desenvolvimento adequado destes. Tem-se que a grande maioria dos criadouros efetua a alimentação de forma manual e altamente 
Citação (APA): Amorim, M. de J., Sousa, G. R., \& Tosta, M. de C. R. (2021). Pesquisa bibliográfica sobre implementação da automação na piscicultura. Brazilian Journal of Production Engineering, 6(8), Edição Especial "Pesca e Aquicultura: Gestão, Política e Inovação", 85-96.

variável. A produtividade do tanque é reduzida quando as alimentações são realizadas em pontos definidos e em intervalos de tempo periódicos, pois dessa forma, os peixes maiores dominam a área de alimentação e impedem a aproximação dos menores, quando estes tentam receber a ração necessária (Calil, 2005). Assim, é necessário o envolvimento de métodos avançados para controle, tais como os geralmente utilizados sistemas de automação (Nääs, 2011).

Com intuito de aumentar a produtividade e lucratividade, pesquisas foram desenvolvidas para garantir melhor qualidade da água nos ambientes de cultivo, maior eficiência no manejo alimentar, menor desperdício na quantidade de ração, maior homogeneidade dos lotes, bem como a redução dos ciclos (Brito et al., 2017). Cubitt et al. (2008) alcançaram resultados plausíveis em sua pesquisa ao utilizarem sensores implantados na musculatura estomacal dos peixes, com capacidade de informar a existência de alimento no estômago destes, o que possibilitou a indicação para o conhecimento se as trutas estavam saciadas ou com fome.

A maior frequência alimentar pode promover o aumento do crescimento dos peixes (Canton, Weingartner, Fracalossi \& Zaniboni, 2007), como também pode proporcionar benefícios, tal como a melhor distribuição do alimento ao longo do dia, o que resulta em melhor aproveitamento da ração e diminuição de desperdícios (Sousa et al., 2012).

O trabalho desenvolvido por Nwanna, Lemme, Metwally e Schwarz (2012) avaliou três estratégias alimentares para carpas (Cyprinus carpio L.), sendo estas: a alimentação de forma manual, exercida duas vezes ao dia, na qual forneceu a ração até alcançar a saciedade aparente dos animais; a alimentação contínua, com mesma quantidade de ração fornecida em relação ao tratamento manual e por fim, a alimentação contínua por meio do emprego de equipamentos automáticos, com fornecimento de ração na proporção de 1,4\% do peso corporal dos peixes. Assim, as três estratégias influenciaram diretamente no ganho de peso e conversão alimentar das carpas, entretanto, a que apresentou melhor resultado para os parâmetros analisados, foi a estratégia que empregou os equipamentos automáticos no fornecimento de ração na proporção de $1,4 \%$.

Outros estudos também mostraram que a maior frequência alimentar (24, 48 e até 96 vezes ao dia) dos organismos aquáticos faz com que estes apresentem melhor desempenho, maior uniformidade e melhor aproveitamento da dieta ofertada (Castro et al., 2014; Sousa et al., 2012).

Hossain, Haylor e Beveridge (2001), por meio do uso de alimentadores automáticos, avaliaram diferentes períodos para se realizar a alimentação de juvenis do Bagre-africano (Clarias gariepinus). Como resultado, quando o fornecimento do alimento foi realizado no período noturno ou continuamente (dia/noite), houve a melhora na conversão alimentar, aumento no crescimento dos animais, bem como menor desperdício de ração.

Já o estudo realizado por Argentim (2016), este avaliou a influência que a temperatura da água e da frequência alimentar apresenta com relação ao desempenho de juvenis de Bijupirá (Rachycentron canadum), criados em sistema de recirculação de água. Os resultados constataram que houve interação da temperatura da água e frequência de alimentação sobre o desempenho dos peixes. $\mathrm{O}$ autor ainda realizou a avaliação do controle automatizado da oferta 
Citação (APA): Amorim, M. de J., Sousa, G. R., \& Tosta, M. de C. R. (2021). Pesquisa bibliográfica sobre implementação da automação na piscicultura. Brazilian Journal of Production Engineering, 6(8), Edição Especial "Pesca e Aquicultura: Gestão, Política e Inovação", 85-96.

da ração conforme a temperatura da água, bem como avaliou os diferentes manejos de alimentação no cultivo de bijupirá, onde concluiu que a alta frequência alimentar (24 vezes/dia) proporcionou melhor aproveitamento da ração pelos animais, como também seu maior crescimento.

\section{AutomaÇão na Piscicultura}

Por meio do controle da qualidade da água é possível aumentar a produtividade da piscicultura e determinar o sucesso ou fracasso do empreendimento (Calil, 2005). Além disso, alinhado ao fato dos peixes se tratarem de animais pecilotérmicos, isto é, são animais de "sangue frio", eles são afetados pelo ambiente externo (Cyrino, Bicudo, Sado, Borghesi \& Dairik, 2010).

Isto posto, a qualidade da água influencia diretamente no desenvolvimento e comportamento do animal. Para garantir bons índices de produtividade é necessário realizar o controle do ambiente onde se cria o indivíduo. Ademais, a sua alimentação varia com a temperatura, quando esta se encontra alta, o consumo de ração é maior (Ostrensky \& Boeger, 1998).

Nessa conjuntura a automação auxilia no controle e monitoramento dos fatores que influenciam na qualidade da água, sendo os principais: temperatura, oxigênio e $\mathrm{pH}$. Desta forma, é possível tirar melhor proveito produtivo do peixe (Brito et al., 2017). A seguir serão abordados os principais protótipos levantados pela revisão para auxiliar neste controle e monitoramento.

\subsection{Protótipos}

Nesta seção serão apresentados os principais modelos desenvolvidos, conforme o levantamento realizado neste estudo, para realizar automação na piscicultura e como estes podem influenciar na mesma.

\subsubsection{Arduino}

Por meio dos protótipos que utilizam Arduino, é possível desenvolver um alimentador automático, no qual permite que a alimentação seja fracionada e pré-programada. Este é constituído por reservatório de ração, motor-redutor, bateria, conector, tubo principal, rosca transportadora, base flutuante e um sistema de alimentação automática controlado por uma plataforma micro controlada, Arduino Uno ${ }^{\circledR}$ e módulo relé (Anschau, 2016).

Ainda, estes modelos de protótipos são capazes de verificar a temperatura, oxigênio dissolvido e $\mathrm{pH}$, sendo estes indicadores que necessitam de monitoração diária, tendo em vista sua importância como parâmetro para qualidade da água (Zaccharias \& Rocha, 2016). Na Figura 1, são identificados os componentes utilizados no projeto. 
Figura 1. Protótipo em Arduino

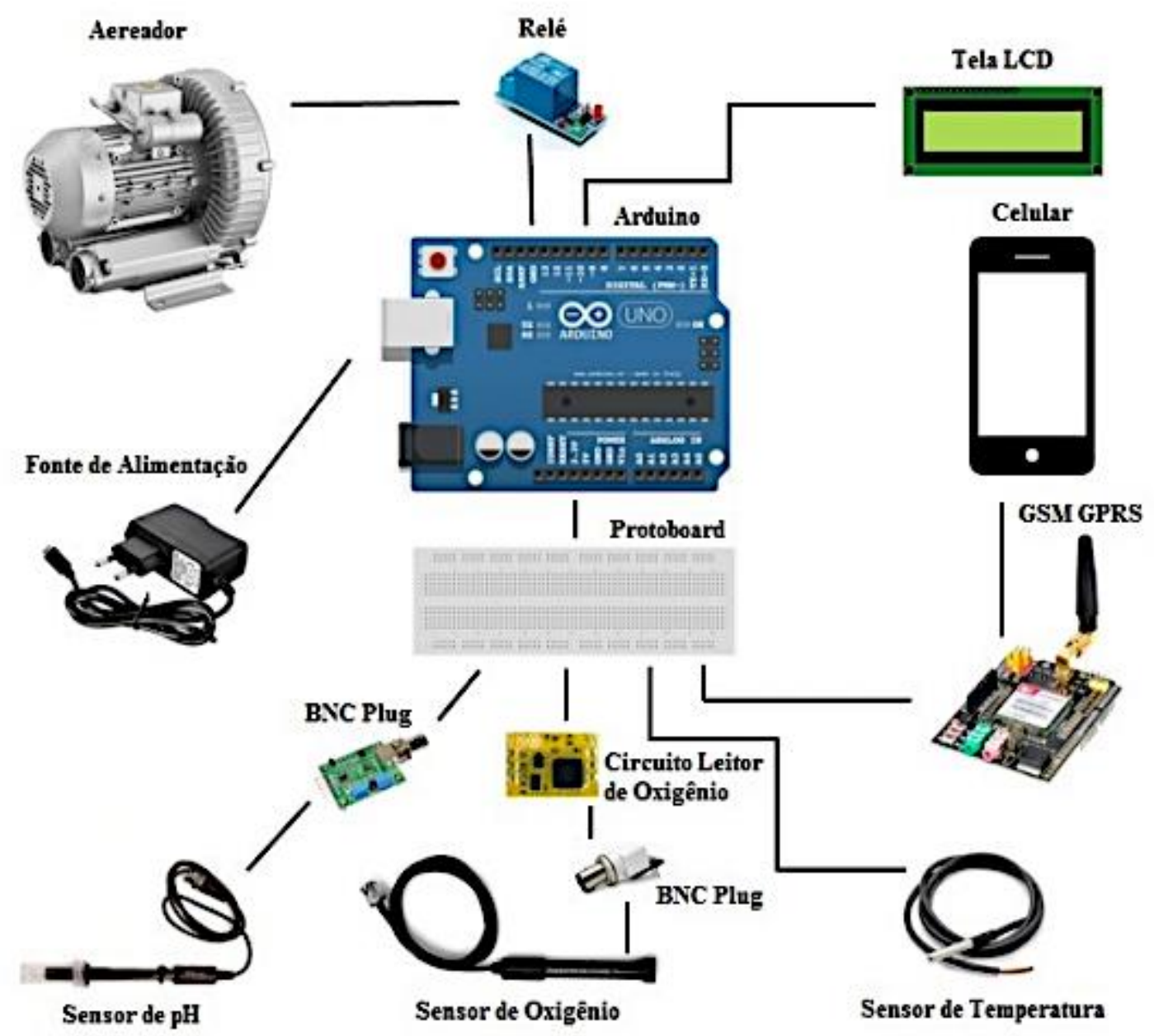

Fonte: Zaccharias \& Rocha (2016).

Com relação às especificações do projeto, os sensores responsáveis pelo monitoramento dos parâmetros possuem limites de medições. No Quadro 1 é apresentada a relação entre os sensores utilizados em cada parâmetro e suas especificações:

Quadro 1. Especificação dos sensores

\begin{tabular}{|c|c|c|}
\hline Parâmetro & Sensores & Especificações \\
\hline Temperatura & $\begin{array}{l}\text { Sensor de temperatura } \\
\text { DS } 18 \mathrm{~B} 20\end{array}$ & Monitora temperaturas em escala Celsius entre $-55^{\circ}$ até $+125^{\circ}$ \\
\hline $\begin{array}{l}\text { Oxigênio } \\
\text { dissolvido }\end{array}$ & Atlas Scientific & $\begin{array}{c}\text { Identifica o oxigênio em uma escala de } 0 \text { a } 35 \mathrm{mg} / \mathrm{L} \text {, pode } \\
\text { atuar a até } 60 \text { metros de profundidade }\end{array}$ \\
\hline $\mathrm{pH}$ & $\begin{array}{l}\text { Eletrodo de } \mathrm{pH} \text { com } \\
\text { conector BNC }\end{array}$ & $\begin{array}{c}\text { Escala relacionada à concentração de íons de hidrogênio } \\
\text { variando de } 0 \text { a } 14\end{array}$ \\
\hline
\end{tabular}

Fonte: Autores (2020).

Após finalizar a construção e programação do protótipo, é necessário inserir em quais condições os parâmetros estarão em boas condições. Deste modo, o sistema executará medidas 
Citação (APA): Amorim, M. de J., Sousa, G. R., \& Tosta, M. de C. R. (2021). Pesquisa bibliográfica sobre implementação da automação na piscicultura. Brazilian Journal of Production Engineering, 6(8), Edição Especial "Pesca e Aquicultura: Gestão, Política e Inovação", 85-96.

preestabelecidas de acordo com as necessidades da espécie que os produtores estão cultivando (Zaccharias \& Rocha, 2016).

Ao obter os dados aferidos pelos sensores, e diante da leitura de alguma irregularidade nas medidas aceitáveis de sobrevivência dos peixes, o sistema poderá agir de forma autônoma na solução do problema, ou então informar ao piscicultor que será necessário intervir para que os níveis aferidos voltem à normalidade.

Portanto a principal função deste projeto é de identificar irregularidades nas medidas de sobrevivência dos peixes, podendo este notificar o produtor sobre algum problema com as condições do ambiente onde o peixe está inserido ou até mesmo agir de forma automática na solução do problema, realizando a correção da temperatura, $\mathrm{pH}$ e/ou oxigênio dissolvido.

\subsubsection{Microprocessador ATMEL modelo ATmega8}

Os sensores utilizados no projeto de automação de um aquário para piscicultura desenvolvido por Beck, Silva, Guerra e Messias (2006) foram selecionados tendo-se em vista a facilidade para aquisição e o baixo custo. Dessa forma, ao que tange ao parâmetro temperatura, utilizouse um semicondutor (LM35). Quanto ao pH, o sensor escolhido é um eletrodo do modelo EPC70 da Instrutherm. Com relação a turbidez da água, essa é mensurada por meio de um sistema de fotodiodo, que indica o nível de sua transparência, sendo um sistema simples e de baixo custo. Com relação ao nível de água, este possui seu controle realizado por meio de um sensor de pressão da família MPX da Freescale Semiconductors.

Para o processamento, foi escolhido para o projeto o microprocessador da Atmel, modelo ATmega8, por apresentar baixo custo e possuir simples programação, esta realizada em linguagem C. A leitura dos sinais emitidos pelos sensores é realizada pelo programa, de acordo com a necessidade de atuação para controle das variáveis.

Quanto ao mais, a composição do mecanismo desenvolvido pelos autores é feita por um micromotor redutor, placas acrílicas e um reservatório para armazenar a comida necessária para a alimentação dos animais.

\subsubsection{Controlador Lógico Programável - CLP}

Agostinho et al. (2014) propuseram a utilização dos CLP (Controladores Lógicos Programáveis) para a realização do controle de alimentadores automáticos para peixes e rãs, de modo que a correção diária do fornecimento fosse realizada conforme o crescimento diário e ajuste instantâneo da quantidade de ração, considerando os parâmetros temperatura e oxigênio dissolvido na água. Para tal, os autores desenvolveram um programa para CLP (Aqui-O-Matic), no qual este é carregado no CLP, de modo a conter as rotinas necessárias para os intervalos de tempo no fornecimento de ração, bem como realizar correções, conforme a variação nos parâmetros da água, realizado por intermédio de sensores de temperatura e oxigênio, conectados a entrada do CLP.

Isto posto, no Quadro 2 é observada a comparação entre os controladores supracitados, ao que tange suas respectivas funções e custos, estes baseados em preços verificados através do Mercado Livre (n.d.). 
Citação (APA): Amorim, M. de J., Sousa, G. R., \& Tosta, M. de C. R. (2021). Pesquisa bibliográfica sobre implementação da automação na piscicultura. Brazilian Journal of Production Engineering, 6(8), Edição Especial "Pesca e Aquicultura: Gestão, Política e Inovação", 85-96.

Quadro 2. Relação entre funções e custos de cada controlador

\begin{tabular}{|c|c|c|}
\hline Função & Controlador & Custo \\
\hline $\begin{array}{c}\text { Desenvolver alimentador automático; verificar temperatura, } \\
\text { oxigênio dissolvido e pH por intermédio de sensores }\end{array}$ & Arduino UNO & $\begin{array}{c}\text { Baixo } \\
\text { custo }\end{array}$ \\
\hline $\begin{array}{c}\text { Realizar o controle das variáveis, como temperatura, pH, } \\
\text { turbidez da água, nível de água por intermédio de sensores }\end{array}$ & $\begin{array}{c}\text { Microprocessador ATMEL } \\
\text { modelo ATmega8 }\end{array}$ & $\begin{array}{c}\text { Baixo } \\
\text { custo }\end{array}$ \\
\hline $\begin{array}{c}\text { Controlar alimentadores automáticos, considerando variação de } \\
\text { temperatura e oxigênio dissolvido por intermédio de sensores }\end{array}$ & $\begin{array}{c}\text { Controlador Lógico } \\
\text { Programável - CLP }\end{array}$ & $\begin{array}{c}\text { Elevado } \\
\text { custo }\end{array}$ \\
\hline
\end{tabular}

Fonte: Arduino (2021); Beck et al. (2006); Agostinho et al. (2014).

\subsection{EMPRESAS}

A seguir serão apresentados os principais meios de automatização na piscicultura, desenvolvidos por empresas que prestam tal serviço.

\subsubsection{Plataforma de automação}

Algumas empresas fornecem uma plataforma para automatização da piscicultura, sendo assim dispensada a necessidade de desenvolver um equipamento por parte do produtor para realizar, por exemplo, alimentador automático, monitoramento dos principais parâmetros da água na piscicultura.

A empresa TatilFish (s.d.) oferece automatização no controle dos alimentos e monitoramento da concentração de oxigênio dissolvido, temperatura, utilizando respectivamente controle automático de aeração e de arraçoamento. Além disso, os equipamentos são alugados ao produtor, o que diminui os custos iniciais de implementação.

Ainda, segundo a empresa, a implementação ocorre por meio da instalação de uma estação de monitoramento, na qual são coletados os dados relacionados ao $\mathrm{pH}$, temperatura e oxigênio dissolvido, em seguida é instalado um controle de automação que monitora e controla o funcionamento dos equipamentos.

Outra empresa do ramo, a Acqua Nativa (2018) oferece soluções e tecnologia para monitoramento da piscicultura, por meio de eletrodos. Em seu portfólio são encontrados os medidores AcquaMeter-AP portátil de multiparâmetros, que permite leituras de até 10 parâmetros simultâneos. Este é compatível com a sonda multiparâmetros AcquaProbe. Ainda, por meio deste é possível monitorar com precisão e em tempo real o oxigênio dissolvido, ORP, $\mathrm{pH}$, condutividade elétrica, salinidade, Íons Seletivos (como amônia e nitrito), TDS e temperatura.

O AcquaLogger-AP é um controlador, medidor e datalogger industrial multiparâmetros. Suporta a leitura de até 4 parâmetros, como oxigênio dissolvido, nitrito, amônia, pH e temperatura. Além disso, por meio deste é possível realizar o monitoramento remoto da qualidade da água e enviar constantemente informações via GRPS, radiofrequência ou por comunicação serial EIA-485.

Ademais, a possibilidade da compra dos equipamentos separadamente, sendo alguns destes: aeradores, alimentadores, incubadoras, selecionadores, medidores, bombas, acessórios. 
Citação (APA): Amorim, M. de J., Sousa, G. R., \& Tosta, M. de C. R. (2021). Pesquisa bibliográfica sobre implementação da automação na piscicultura. Brazilian Journal of Production Engineering, 6(8), Edição Especial "Pesca e Aquicultura: Gestão, Política e Inovação", 85-96.

Optando pela compra separada destes equipamentos, o produtor consegue automatizar a sua produção gradualmente, conforme sua opção de investimento.

Algumas empresas oferecem estações de piscicultura, sendo este o caso da Sigma Sensores (s.d.) que oferece a Estação Básica Onset para Piscicultura com Telemetria de Dados, sendo esta uma estação automatizada que envia de 10 em 10 minutos os dados coletados em campo, para isso utiliza-se telemetria, através dos sinais de rede (GSM / WIFI / ETH ou SATÉLITE), estes são direcionados para uma plataforma online na internet e gratuita chamada Hobolink.

\section{CONSIDERAÇÕES FINAIS}

Os piscicultores possuem diversos meios para implementar automação em sua produção, partindo desde a utilização dos projetos de menor custo, por exemplo, Arduino UNO e o microprocessador ATMEL modelo ATmega8 até empresas que realizam automação completa ou parcial do processo produtivo.

Desta forma, o produtor consegue modernizar a sua empresa e melhorar a produtividade, já que a qualidade da água influencia diretamente no desenvolvimento e comportamento do animal. Para garantir bons índices de produtividade é necessário realizar o controle do ambiente onde se cria o indivíduo, e para isso, deve-se monitorar parâmetros como temperatura, oxigênio dissolvido e $\mathrm{pH}$ da água.

Ainda, a alimentação do peixe requer uma atenção, sendo de grande importância para a produção, uma vez que a ração de qualidade é fundamental para os animais se desenvolverem corretamente. Ademais, a alimentação é um dos problemas mais graves da criação de peixes em tanques artificiais, pois essa precisa ser equilibrada e na quantidade correta para o desenvolvimento adequado destes. Para isso, por meio da automação existem os alimentadores automáticos, os quais permitem que a alimentação seja fracionada e pré-programada.

Isto posto, existem diversos meios para automatizar a produção na piscicultura, ficando ao piscicultor a escolha do modelo que melhor se encaixa a sua produção e ao investimento possível de acordo com sua realidade, pois este processo possui implementações de baixo a alto custo. Por fim, cabe salientar que, assim como em outros setores da indústria, a modernização da linha de produção na piscicultura se faz cada vez mais presente e necessária.

\section{REFERÊNCIAS}

Acqua Nativa. (2018). Monitoramento em tempo real de cargas e viveiros na piscicultura: $p H$, nitrogênio, oxigênio dissolvido e temperatura. Recuperado de https://www.acquanativa.com.br/aplicacoes/monitoramento-piscicultura-tempo-real.html

Agência Brasileira de Desenvolvimento Industrial. (n.d.). Agenda brasileira para a Indústria 4.0. Recuperado de http://www.industria40.gov.br/

Agostinho, C.A. et al. (2014). Aqui o Matic: Programa para a automação do fornecimento de ração para peixes e rãs com base nas variações da temperatura da água, no oxigênio dissolvido e no ganho diário de peso estimado com base na conversão esperada. INPI - Instituto Nacional da Propriedade Industrial. 
Citação (APA): Amorim, M. de J., Sousa, G. R., \& Tosta, M. de C. R. (2021). Pesquisa bibliográfica sobre implementação da automação na piscicultura. Brazilian Journal of Production Engineering, 6(8), Edição Especial "Pesca e Aquicultura: Gestão, Política e Inovação", 85-96.

Anschau, S. P. (2016). Protótipo de alimentador automático para a larvicultura da tilápia (Oreochromis niloticus)(Dissertação de mestrado). Universidade Estadual do Oeste do Paraná, Toledo, PR, Brasil.

Arduino. (2021). $O$ que é Arduino? Recuperado de https://www.arduino.cc/en/Guide/Introduction

Argentim, D. (2016). Automação do manejo alimentar de bijupirá (Rachycentron canadum) (Dissertação de doutorado). Universidade Estadual Paulista, Botucatu, SP, Brasil.

Beck, J.C., Silva, I.N., Guerra, K., \& Messias, D.E. (2006). Automação e controle de tanque para Piscicultura. Anais do XXXIV COBENGE. Passo Fundo, RS, Brasil.

Brettel, M., Friederichsen, N., Keller, M., \& Rosenberg, M. (2014). How Virtualization, Decentralization and Network Building Change the Manufacturing Landscape: An Industry 4.0 Perspective. International Scholarly and Scientific Research \& Innovation, London, 8(1), 3744.

Brito, J. M., Pontes, T. C., Tsujii, K. M., Araújo, F. E., \& Ricther, B. L. (2017). Automação na tilapicultura: revisão de literatura, desempenho, piscicultura, tecnologias, tilápias. Nutritime, 14 (3), 5053-5062.

Calil, B.M. (2005). Automação de Piscicultura em Tanques Artificiais (Dissertação de mestrado). Universidade de Taubaté, Taubaté, SP, Brasil.

Canton, R., Weingartner, M., Fracalossi, D.M., \& Zaniboni, E., Filho. (2007). Influência da frequência alimentar no desempenho de juvenis de jundiá. Revista Brasileira de Zootecnia, Viçosa, 36 (4), 749-753.

Castro, C.S., Ribeiro, R.R., Agostinho, L.M., Santos, A.A.D., Carmelin, C.A., Jr., Chan, R.V., ... \& Agostinho, C.A. (2014). Polyculture of frogs and tilapia in cages with high feeding frequency. Aquacultural Engineering, Amsterdam, 61, 43-48.

Cyrino, J.E.P., Bicudo, Á.J.D.A., Sado, R.Y., Borghesi, R., \& Dairik, J.K. (2010). A Piscicultura e o ambiente - o uso de alimentos ambientalmente corretos em piscicultura. Revista Brasileira de Zootecnia, São Paulo, 39, 68-87.

Danoesastro, M.; Freeland, G.; \& Reichert, T. (2017). A CEO's guide to leading digital transformation. $B C G$ - Perspectives, Boston, 1-4.

Cubitt, K.F., Williams, H.T., Rowsell, D., McFarlane, W.J., Gosine, R.G., Butterworth, K.G. \& McKinley, R.S. (2008). Development of an intelligent reasoning system to distinguish hunger states in Rainbow trout (Oncorhynchus mykiss). Computers and Electronics in Agriculture, Amsterdam, 62(1), 29-34.

Fleury, A.C.C. (1978). Organização do trabalho industrial: um confronto entre teoria e realidade (Dissertação de doutorado). Escola Politécnica da Universidade de São Paulo, São Paulo, SP, Brasil.

Hossain, M.A.R., Haylor, G.S., \& Beveridge, M.C.M. (2001). Effect of feeding time and frequency on the growth and feed utilization of African catfish Clarias gariepinus (Burchell, 1822) fingerlings. Aquaculture Research, Chichester, 32, 999-1004.

Kubitza, F. (2019). Alimentação automatizada na aquicultura: ganhos em eficiência e redução de custos. Panorama da aquicultura, 29(171), 20-21.

Lekang, O. (2009). Aquaculture engineering. Blackwell Publishing Ltd.

Longo, W.P. (1984). Tecnologia e soberania nacional. Nobel.

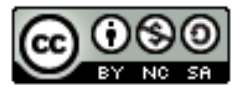


Citação (APA): Amorim, M. de J., Sousa, G. R., \& Tosta, M. de C. R. (2021). Pesquisa bibliográfica sobre implementação da automação na piscicultura. Brazilian Journal of Production Engineering, 6(8), Edição Especial "Pesca e Aquicultura: Gestão, Política e Inovação", 85-96.

Maciente, A.N.; Rauen, C. V.; \& Kubota, L. C. (2019). Tecnologias digitais, habilidades ocupacionais e emprego formal no Brasil entre 2003 e 2017. Mercado de Trabalho: conjuntura e análise, 66, 115-129.

Matos, J.S. (2018). A Indústria 4.0 na economia brasileira: Seus benefícios, impactos e desafios (Trabalho de monografia). Universidade Federal de Uberlândia, Uberlândia, MG, Brasil.

Mercado Livre. (n.d.) Arduino uno. Recuperado de https://lista.mercadolivre.com.br/arduinouno

Mercado Livre. (n.d.). Controladores lógicos programáveis. Recuperado de https://lista.mercadolivre.com.br/controladores-logicos-programaveis

Mercado Livre. (n.d.) Microprocessador atmel modelo atmega8. Recuperado de https://lista.mercadolivre.com.br/microprocessador-atmel-modelo-atmega8

Nääs, I.A. (2011). Uso de técnicas de precisão na produção animal. Revista Brasileira de Zootecnia, 40, 358-364.

Nwanna, L.C., Lemme, A., Metwally, A., \& Schwarz, F.J. (2012). Response of common carp (Cyprinus carpio L.) to supplemental DLmethionine and different feeding strategies. Aquaculture, 356, 365-370.

Ostrensky, A., \& Boeger, V. (1998). Piscicultura: Fundamentos e Técnicas de Manejo. Guaíba: Agropecuária.

Schulter, E.P.; \& Vieira, J.E.R., Filho. (2017). Evolução da piscicultura no brasil: diagnóstico e desenvolvimento da cadeia produtiva de tilápia [Texto para discussão, $\mathrm{N}^{\circ} 2328$ ]. Rio de Janeiro, RJ: IPEA.

Schwab, K. (2017). The fourth industrial revolution. New York: Crown Business.

Sigma Sensores. (s.d.). Estações para Piscicultura com Telemetria de Dados. Recuperado de https://sigmasensors.com.br/estacao-piscicultura

Siqueira, T.V. (2018). Aquicultura: a nova fronteira para produção de alimentos de forma sustentável. Revista do BNDES, 25(49), 119-170.

Sousa, R.M.R., Agostinho, C.A., Oliveira, F.A., Argentim, D., Novelli, P.K., \& Agostinho, S.M. M. (2012). Productive performance of Nile tilapia (Oreochromis niloticus) fed at different frequencies and periods with automatic dispenser. Arquivo Brasileiro de Medicina Veterinária e Zootecnia, Belo Horizonte, 64 (1), 192-197.

Suzuki, M.A.A., Hernandez, F.B.T. (1999). Automação de sistemas de irrigação. Ilha Solteira: Faculdade de Engenharia de Ilha Solteira - UNESP.

TatilFish. (s.d.). A TATILFish auxilia o produtor a obter Sucesso na Piscicultura. Recuperado de https://www.tatilfish.com.br/

Trabachini, A. (2013). Sistema automatizado de alimentação automatizada para suínos visando aplicação em rastreabilidade animal. (Dissertação de mestrado). Universidade de São Paulo, Piracicaba, SP, Brasil.

Zaccharias, R.L., \& Rocha, R.V. (2016). Automação dos processos de produção e controle para aumento de produtividade e redução de desperdícios na piscicultura. Revista Eletrônica Competências Digitais para Agricultura Familiar, 2(2), 52-67.

Zion, B. (2012). The use of computer vision technologies in aquaculture - A review. Computers and Electronics in Agriculture, 88, 125-132. 